Research Article

\title{
On Credit Risk Contagion of Supply Chain Finance under COVID-19
}

\author{
Wentao Chen $\mathbb{D}^{1},{ }^{1}$ Zhenlin Li $\mathbb{D}^{1},{ }^{1}$ and Zhuoxin Xiao $\mathbb{D}^{2}$ \\ ${ }^{1}$ College of Finance and Statistics, Hunan University, Changsha, China \\ ${ }^{2}$ China Development Bank, Beijing, China \\ Correspondence should be addressed to Zhenlin Li; zhenlinlee@hnu.edu.cn
}

Received 2 September 2021; Accepted 13 September 2021; Published 28 September 2021

Academic Editor: Sun Young Cho

Copyright ( $\odot 2021$ Wentao Chen et al. This is an open access article distributed under the Creative Commons Attribution License, which permits unrestricted use, distribution, and reproduction in any medium, provided the original work is properly cited.

Existing research on credit risk contagion of supply chain finance pays more attention to the influence of network internal structure on the process of risk contagion. The spread of COVID-19 has had a huge impact on the supply chain, with a large number of enterprises experiencing difficulties in operation, resulting in increased credit risks in supply chain finance. Under the impact of the epidemic, this paper explores the transmission speed and steady state of credit risk when the supply chain finance network is affected by external impact so that we can have a more complete understanding of the ability of supply chain finance to resist risks. The simulation results show that external shocks of different degrees will increase the number of initial infected enterprises and lead to the increase in credit risk contagion speed but have no significant impact on network steady state; the speed of credit risk contagion is positively correlated with network complexity but not significantly affected by network size; core enterprises infected will increase the rate of credit risk contagion. The intensity of policy intervention has obvious curative effect on the risk caused by external shock. When the supply chain financial network is affected by external shocks, the intensity, time, and pertinence of policy response can effectively prevent the credit risk contagion.

\section{Introduction}

Since the outbreak in 2020, COVID-19 has spread to 191 countries and regions, with more than 200 million confirmed cases and 4.4 million deaths. As a result of the epidemic, the unemployment rate has gradually increased, enterprises have suspended production, and economic indicators have declined significantly. Optimization methods, in particular, fixed-point methods, are efficient and powerful for solving various real problems in traffic and transportation, medical imaging, modern management, financial engineering (see, e.g., [1-5]). For the supply chain, the continuous decline of market demand and production stagnation of upstream and downstream enterprises will lead to serious operational difficulties for enterprises within the supply chain. Repeated outbreaks caused by mutations in the virus have cast a shadow over the recovery of global supply chain. Against the backdrop of the pandemic, supply chain finance is also facing severe impact.
Supply chain finance integrates logistics, capital flow, and information flow and forms a corresponding network structure. With the continuous advancement of financial globalization, network complexity is strengthened [6], while making the network more efficient, it also makes it more vulnerable to default and bankruptcy [7]. The credit default of one participant will cause losses of other members and eventually lead to credit risk contagion throughout the supply chain network [8-11]. The spread of COVID-19 has significantly increased the network credit risk of supply chain finance. The supply chain finance plays a role in resisting the external impact and effectively offsetting losses caused by the COVID-19 outbreak; therefore, it is important to study the influence of external impact on supply chain financial credit risk infection and corresponding policies.

The rest of this paper is organized as follows. Section 2 is literature review, which mainly summarizes the existing research on credit risk of supply chain finance. Section 3 is the construction of complex network of supply chain 
finance, including model selection and model construction. Section 4 is the simulation results and discussion, the design of the relevant simulation experiments, and the results of indepth analysis. Section 5 is the conclusion.

\section{Literature Review}

Supply chain finance is different from traditional financing methods. By integrating the financing process of all participants and optimizing the capital flow in the supply chain, supply chain finance improves the overall capital management level of the supply chain [12-14]. The existing research on credit risk of supply chain finance mainly focuses on risk identification and evaluation [8], and the research method is mainly based around the construction of the risk evaluation index system. Mou et al. [9] believed that core enterprises are crucial to the evaluation of credit risks in the supply chain, and the FAHP method is used to quantitatively measure and evaluate the credit risks of core enterprises. Zhu et al. [15] believed that SMEs are in a vulnerable position in the supply chain, but their credit risk status is of great significance to supply chain finance. With the help of RS-multiboosting machine learning model, they built a prediction model of credit risk for SMEs in supply chain finance, which has a good effect. Wang et al. [16] believed that the development of smart city and smart finance makes the financial risks of SMEs more complex and uses the improved PROMETHEE method to build a more accurate credit risk evaluation model of SMEs. However, enterprises in the supply chain do not operate in isolation, and the credit risk within the supply chain is bound to infect the whole supply chain, causing serious impact on the network.

On the topic of credit risk contagion, existing research mainly focuses on the influence of internal structure of supply chain on the process of credit risk contagion. Zhao et al. [17] established a scale-free complex network model of supply chain finance and found that network structure had a significant impact on credit risk contagion. Xie et al. [18] made a game analysis of the two-tier supply chain with financing constraints by constructing two-tier financing channels in the supply chain and found that financing structure has a significant impact on credit risk contagion, while the contagion effect under dual-channel financing mechanism is weak. With the help of the SIRS infectious disease model, Wang et al. [11] deeply discussed the influence of multiple factors on supply chain risk contagion, including enterprise risk preference, operational robustness and flexibility, completeness of market information, especially network topology. However, in addition to internal factors, external shocks also have an impact on the process of credit risk contagion. Therefore, this paper mainly studies the impact of external shocks (COVID-19) on the credit risk contagion process of supply chain finance.

\section{Construction of Supply Chain Finance Network}

3.1. Selection of Network Model and Infection Model. From the perspective of the development history of complex network, complex network can be divided into regular network, random graph, small world network, and scale-free network. A regular network is one in which any two nodes are directly connected by an edge; random graph refers to the network formed by connecting nodes randomly according to probability. Both small world network and random network conform to Poisson distribution, which results that the degree of a large number of nodes in the network is concentrated near $k$, and there are no points with a relatively high degree. This kind of network is also called uniform network. In the structure of supply chain finance network, newly joined SMEs tend to be connected with core enterprises with high degree, which makes core enterprises have a very high degree, while SMEs have a relatively low degree. This characteristic of supply chain finance network is exactly in line with the characteristics of scale-free network. Therefore, BA scale-free network is selected as the basis to construct supply chain finance network.

The premise of virus transmission is a network environment conducive to transmission, and only mutual contact and relationship can complete the transmission process, which is consistent with the transmission of credit risk. Therefore, the epidemic model has been widely used to study the transmission of credit risk. The SIS virus infection model is a classical model of transmission dynamics in complex networks and has been applied in a large number of studies on credit risk $[17,19]$. The SIS virus infection model has the advantage that it has no limitation on the network scale and no special requirements on the network transmission direction and can accurately reflect the dynamic process of credit risk infection in the network. Therefore, the SIS virus infection model is very suitable for this paper as the basic model of credit risk infection.

\subsection{Construction of Complex Network of Supply Chain} Finance. Based on the discussion of model selection in the previous section and the special requirements of the network model in related studies $[17,19]$, this paper proposes the following hypotheses for the supply chain finance network:

(i) Hypothesis 1. Supply chain finance network is an undirected network. Generally, risk contagion is transmitted from upstream to downstream, but for supply chain finance network, it is mainly manifested as upstream and downstream cross infection, and there is no clear direction. Therefore, this article sets the network as an undirected network.

(ii) Hypothesis 2. Nodes in the network are only infected by neighboring nodes. In the supply chain finance network, the connection between two nodes is the channel of risk contagion, and nodes without direct contact have little influence on each other, which is not considered in this paper.

(iii) Hypothesis 3. Credit risk in supply chain finance network transmits with a certain probability. Even if it is connected to the infected node, it does not necessarily mean that it will be affected accordingly. The probability of infection is related to the strength of the connection between the two nodes. In this 
paper, to simplify the infection model, this probability is set as a constant.

(iv) Hypothesis 4. The structure and scale of supply chain finance network will not change in the process of credit risk contagion. In the real world, the network is always in a dynamic process, some nodes will join, and also some nodes will disconnect. In this paper, the network model is simplified, and the changes of network structure and scale in the process of infection are not considered.

Assume that the probability of credit risk infection in the network is $\lambda, 0<\lambda<1$; at time $t$, the density of infected individuals on the network is $\rho(t)$. As $t$ approaches infinity, the network reaches a steady state $\rho$. Considering that the scale-free network is a nonuniform network, the infected density of the node with the degree of $k$ at the time of $t$ is $\rho_{k}(t)$. Each node has a certain recovery capability, and the recovery coefficient is set as $\gamma$. Through the comprehensive analysis of the above contents and the characteristics of the SIS model, we can get the following equation at time $t$ :

$$
\frac{\partial \rho_{k}(t)}{\partial t}=-\gamma \rho_{k}(t)+\lambda k\left[1-\rho_{k}(t)\right] \Theta(\rho(t)) .
$$

The first term on the right is the annihilation term, and the density of infected nodes decreases with the speed $\gamma$. The second item is the generation term, and it is proportional to the probability of infection $\lambda$, density of susceptible nodes $\left[1-\rho_{k}(t)\right]$, the degree of node $k$, and $\Theta(\rho(t))$. $\Theta(\rho(t))$ represents the probability that any given edge is connected to an infected node, namely, $\Theta(\rho(t))=(1 /\langle k\rangle) \sum_{k} k P(k) \rho_{k}(t)$. We assume the infected density of the node with degree $k$ at steady state $\rho_{k}$.

Using the steady-state condition $\left(\partial \rho_{k}(t) / \partial t\right)=0$, we have the following:

$$
\rho_{k}=\frac{\lambda k \Theta(\rho)}{\gamma+\lambda k \Theta(\rho)} .
$$

According to equation (2), we find that at steady state, the density of infected nodes with degree $k$ is positively correlated to the infection probability $\lambda$ and the degree of the node $k$ but is negatively correlated to the enterprise's resilience $\gamma$.

With equation (2) and $\Theta(\rho(t))$, we have the following:

$$
\Theta=\frac{1}{\langle k\rangle} \sum_{k} k P(k) \frac{\lambda k \Theta}{\gamma+\lambda k \Theta} .
$$

If the equation has a nontrivial solution $\Theta \neq 0$, the following conditions must be met:

$$
\left.\frac{\mathrm{d}}{\mathrm{d} \Theta}\left(\frac{1}{\langle k\rangle} \sum_{k} k P(k) \frac{\lambda k \Theta}{\gamma+\lambda k \Theta}\right)\right|_{\Theta=0} \geq 1
$$

Namely,

$$
\sum_{k} \frac{\lambda k^{2} P(k)}{\gamma\langle k\rangle}=\frac{\lambda\left\langle k^{2}\right\rangle}{\langle k\rangle} \geq 1 .
$$

The critical value $\lambda_{c}$ of BA scale-free network is as follows:

$$
\lambda_{c}=\frac{\gamma\langle k\rangle}{\left\langle k^{2}\right\rangle}
$$

In BA scale-free network,

$$
\begin{aligned}
\langle k\rangle & =\int_{m}^{\infty} k P(k) \mathrm{d} k=2 m, \\
\left\langle k^{2}\right\rangle & =\sum_{k} k^{2} P(k), \\
P(k) & =2 m^{2} k^{-3} .
\end{aligned}
$$

Assume that the maximum degree in the supply chain finance network is $k_{l}$; as the network goes to infinity, $k_{l}$ tends to $m N^{2}$, so $\left\langle k^{2}\right\rangle \approx 2 m^{2} \ln \left(k_{l} / m\right)$. The critical value $\lambda_{c}$ can be represented as

$$
\lambda_{c}=\frac{\gamma\langle k\rangle}{\left\langle k^{2}\right\rangle}=\frac{\gamma}{2 m \ln N} .
$$

According to equation (8), we find that the critical value $\lambda_{c}$ is negatively correlated with the network structure $m$ and the network size $N$ but is positively correlated to the enterprise's resilience $\gamma$.

\section{Simulation Results and Discussion under Epidemic Impact}

\subsection{Simulation Algorithm Design}

(1) BA scale-free network is constructed. According to the existing research $[17,18,20]$, this paper sets the basic parameters of the network as $N=1000$ and $m=5$.

(2) According to the scope of the epidemic, the initial infected nodes were randomly selected from the network.

(3) The number of newly infected nodes are determined. Assume that the status of the node $i$ is $S(i)$; when the node is infected, $S(i)=1$; if not infected, $S(i)=0$; External impact on node $i$ can be expressed as

$$
\beta(i)=1-(1-\lambda)^{\alpha_{i}},
$$

where $\alpha_{i}=\sum_{j=1}^{N} a_{i j} S_{j}, i \neq j$ indicates the number of infected nodes in adjacent nodes of the node $i, a_{i j}$ indicates the connection status between node $i$ and node $j, a_{i j}=1$ indicates that the two nodes are directly connected; otherwise, they are not adjacent nodes; $\beta(i)$ denotes the probability that node $i$ is infected by at least one neighboring infected node, which can be used to represent its external impact.

The risk threshold $C_{i}$ of node $i$ can be expressed as

$$
C_{i}=\left(\delta+c_{i}\right) \frac{k_{i}}{2 m}
$$

The current assets owned by enterprises have a certain resistance to credit risk, so the resistance of enterprises is expressed as $\delta$, which is set to 0.25 . To represent the differences of enterprises, we introduce random number $c_{i}$ 
$\left(0<c_{i}<1\right)$. If $C_{i}<\beta(i)$, node $i$ is infected. Otherwise, node $i$ is not infected.

\subsection{Analysis of Simulation Results under Epidemic Impact}

4.2.1. Determine the Risk of Contagion $\lambda$. First, we need to determine the probability of risk contagion $\lambda$ matching the credit risk contagion network, which is related to $\lambda_{c}$ in equation (8); when $\lambda>\lambda_{c}$, credit risk becomes contagious; otherwise, credit risk will not be contagious. According to equation (8), we set the initial network state to $N=1000$, $m=5$, and $\gamma=0.2$ and simulated the influence of network parameters $\gamma, m$, and $N$ on $\lambda$. The simulation results are shown in Figure 1.

According to the above simulation results, we found that with the increase in self-healing rate $\gamma, \lambda_{c}$ increased continuously. With different $\gamma, \lambda_{c}$ is between 0.2 and 0.3 . For network complexity $m$, when $m=1$, the density of infected nodes in the steady state is 0 , indicating that there is no risk infection within the network. With the increase in network complexity, $\lambda_{c}$ gradually decreases, while $\lambda_{c}$ has a maximum value of about 0.3 . There is a negative correlation between the network size and $\lambda_{c}$; when $N=200, \lambda_{c}$ is about 0.21 , and when $N=1000, \lambda_{c}$ is about 0.25 . Therefore, in order to ensure that the simulation results are not affected by risk contagion probability, $\lambda$ is set as 0.3 in the subsequent simulation experiments of this paper.

4.2.2. Analysis of Simulation Results of Enterprise SelfHealing Mode under Epidemic Impact. We first consider that in the case of no policy intervention, the enterprise has a certain self-healing rate $\gamma$. That is, the enterprise has a certain probability to recover to a healthy state. Compared with the credit default of an internal enterprise, the outbreak of COVID-19 will have a more serious impact, mainly reflected in the following: a large number of enterprises are faced with shutdown and production, demand decline, labor shortage, and other problems, which leads to a sudden increase in the number of initially infected enterprises. Under the epidemic, the probability of default of core enterprises increases. As core enterprises are connected with a large number of enterprises, default will inevitably have a serious impact on the entire network. Therefore, in the adjustment of the initial network state, we increased the initial number of infected nodes $m_{0}$ to represent the impact caused by the epidemic impact. The initial number of infected nodes indicates the degree to which different industries are affected by the epidemic. Finally, the influence of the number of core enterprises infected on credit risk contagion is discussed. First, we consider the impact of the initial number of infected nodes on the supply chain finance network, and the simulation results are shown in Figure 2.

According to the simulation results, we find that the initial number of infected nodes $m_{0}$ is basically positively correlated with the contagion speed of credit risk. However, for the steady state, the difference between Figures 2(a) and 2(b) is not obvious because the risk contagion threshold of SMEs $C_{i}$ is relatively low. Without policy intervention and increase in the initial number of infected nodes, most nodes will be infected. Now, we will discuss the impact of core enterprises infection on credit risk contagion. The simulation results are shown in Figure 3.

According to the simulation results, it is found that when the initial number of infected nodes is small $\left(m_{0}=10\right)$ and $c=0$, there is a difference between the credit risk contagion rate and the infection rate of core enterprises. With the increase in external shock intensity (initial infection number), the difference of credit risk contagion speed gradually decreases. The main reason for this phenomenon is that the default of core enterprises will affect a large number of smalland medium-sized enterprises, which makes the contagion speed increase rapidly. The increase in initial infection nodes will also increase the transmission speed of credit risks, thus narrowing the gap between $c=0$ and other cases.

Considering the impact of network nature on credit risk contagion, we will next discuss the resistance of network structure to epidemic impact. There are differences between different types of supply chains, which are related to the attributes of each enterprise and the connections between enterprises. Under the impact of the epidemic, it is worth exploring whether different self-healing rates, network complexity, and network size will affect the transmission of credit risk. Here, set the basic network parameters to $\lambda=0.3$, $c=0, N=1000$, and $m=5$. First, we discuss the self-healing ability of enterprises and its impact on credit risk contagion. The simulation results are shown in Figure 4.

According to the simulation results, we find that the enterprise self-healing rate $\gamma$ has a significant impact on steady state and credit risk contagion speed. When $m_{0}=5$ and $\gamma$ greater than 0.4 , the steady state is 0 , and there is no credit risk contagion. With the continuous decrease in $\gamma$, the contagion rate of credit risk gradually increases. When $\gamma=0.3$, the network basically reaches a steady state at 36 steps. When $\gamma=0.1$, it takes only 20 steps to basically reach steady state. When $m_{0}=5$ and $\gamma=0.3$, the steady state is basically maintained at $80 \%$. When $\gamma=0.1$, the final steady state increases to $90 \%$. This conclusion is basically consistent in the face of different degrees of initial shock: even when the initial number of infected nodes reaches 200, the final steady-state difference between $\gamma=0.1$ and $\gamma=0.5$ remains at $10 \%$. It shows that the enterprise self-healing rate has a regulating effect on the final steady state of the network. In order to ensure that the enterprise self-healing rate would not affect the authenticity of other simulation results, the basic value of the enterprise self-healing rate was set as 0.2 .

The impact of network complexity on credit risk contagion under different external shocks is shown in Figure 5. According to the simulation results, we find that network complexity has a significant impact on the rate of credit risk contagion. When $m_{0}=5$, there is no credit risk contagion in the network where $m$ is less than 2. With the increase in $m$, the contagion rate of credit risk keeps increasing. When $m=6$, the network has basically reached steady state in 6 steps. With the increase in external impact, the law is basically consistent: even if $m_{0}=200$, the number of infected nodes at the initial stage of $m=1$ increases rapidly; however, after 4 steps, the number of infected nodes began to decline 


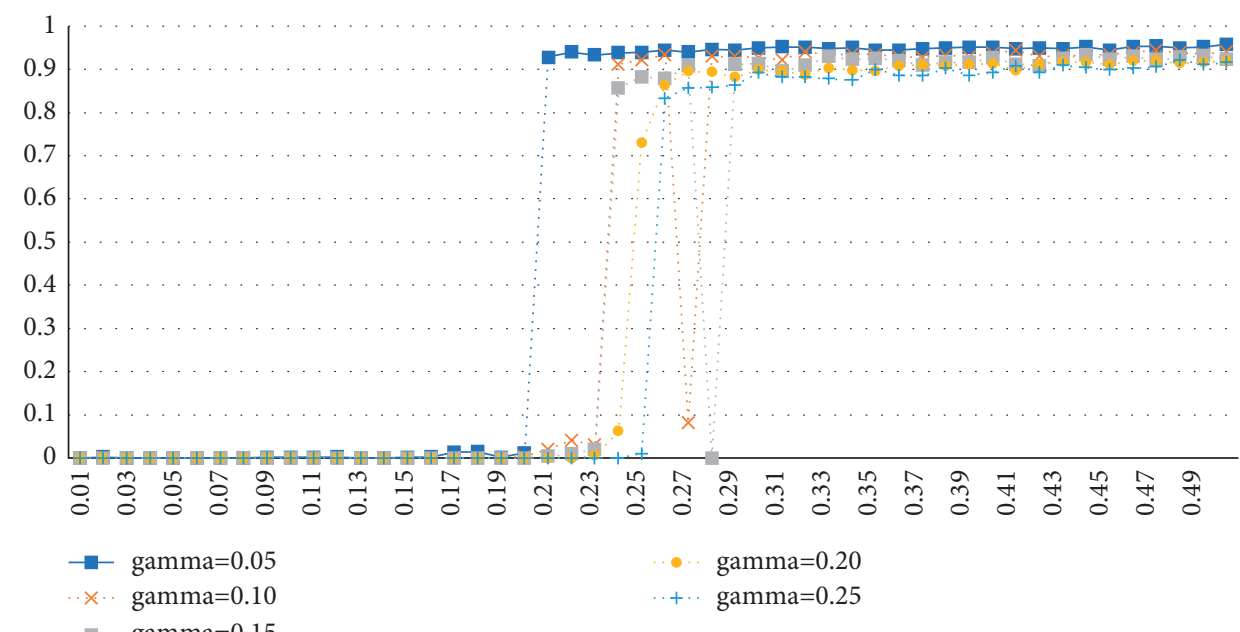

(a)

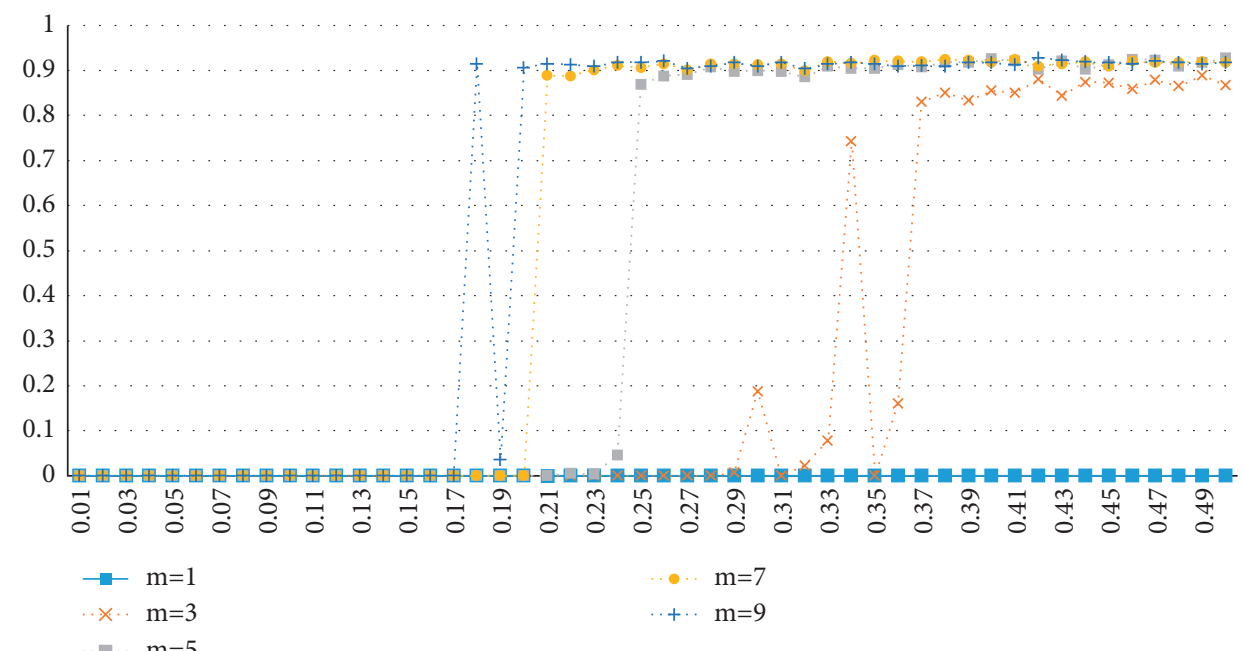

(b)

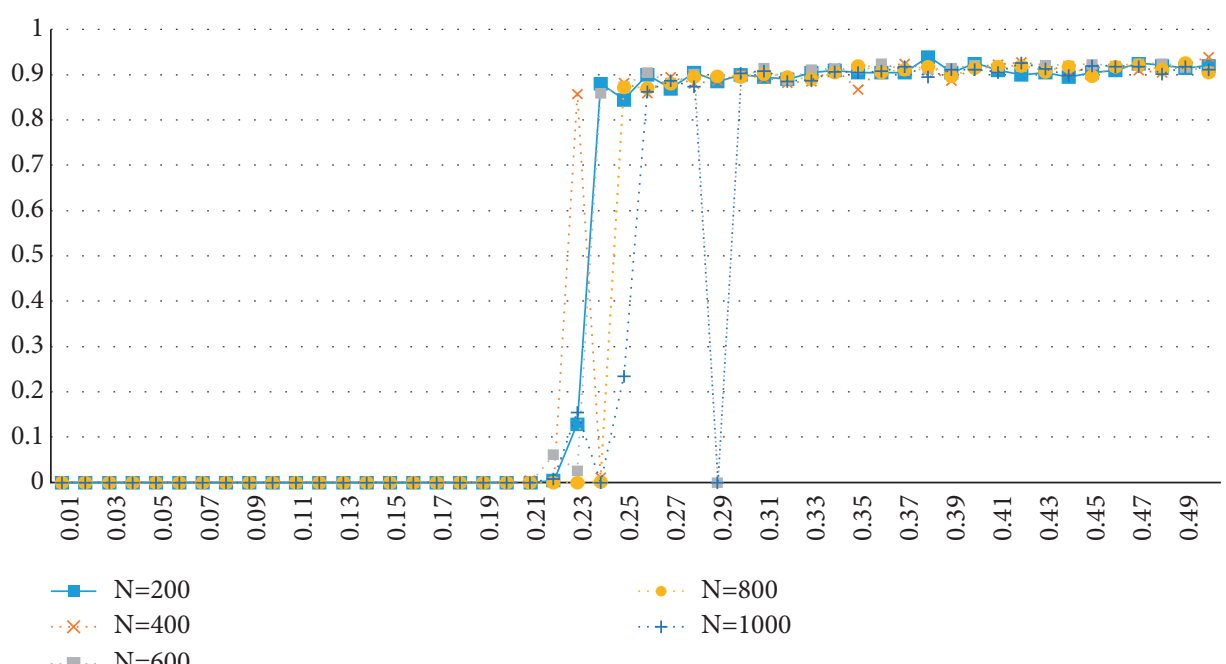

(c)

FIgURE 1: The influence of network parameters on risk contagion probability $\lambda$. (a) $\gamma$ ranges from 0.05 to 0.25 ; step length is 0.05 . (b) $m$ ranges from 1 to 9; step length is 2. (c) $N$ ranges from 200 to 1000; step length is 200. 


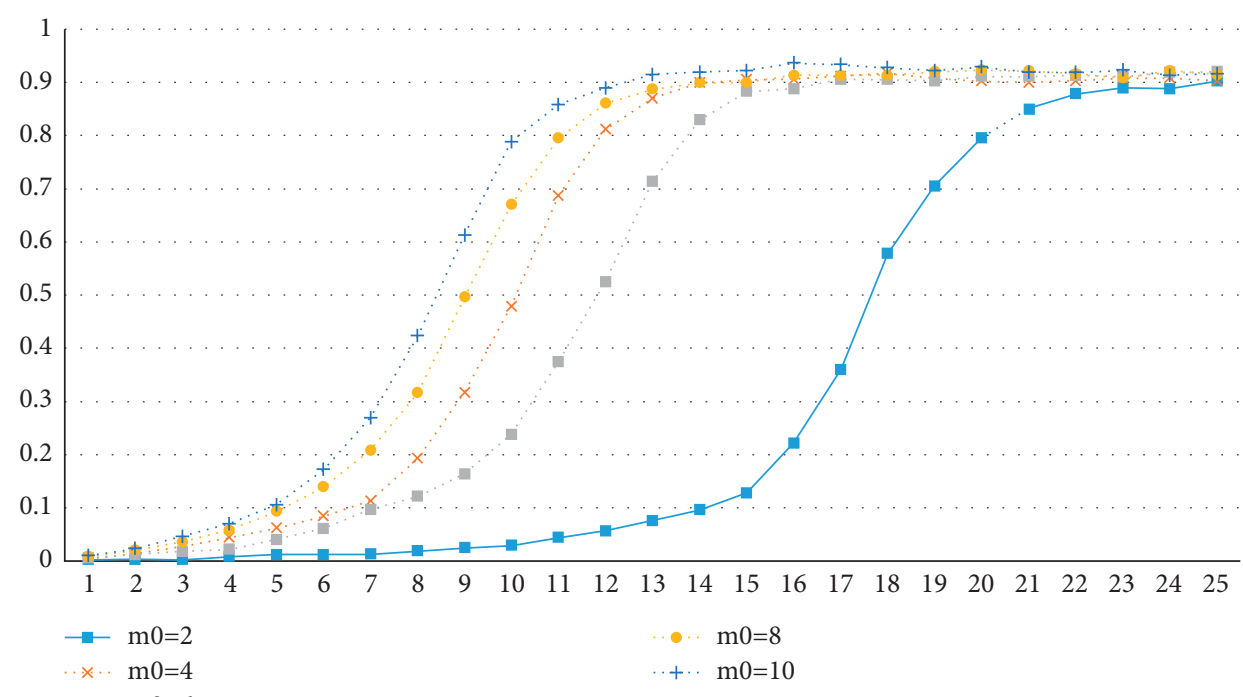

(a)

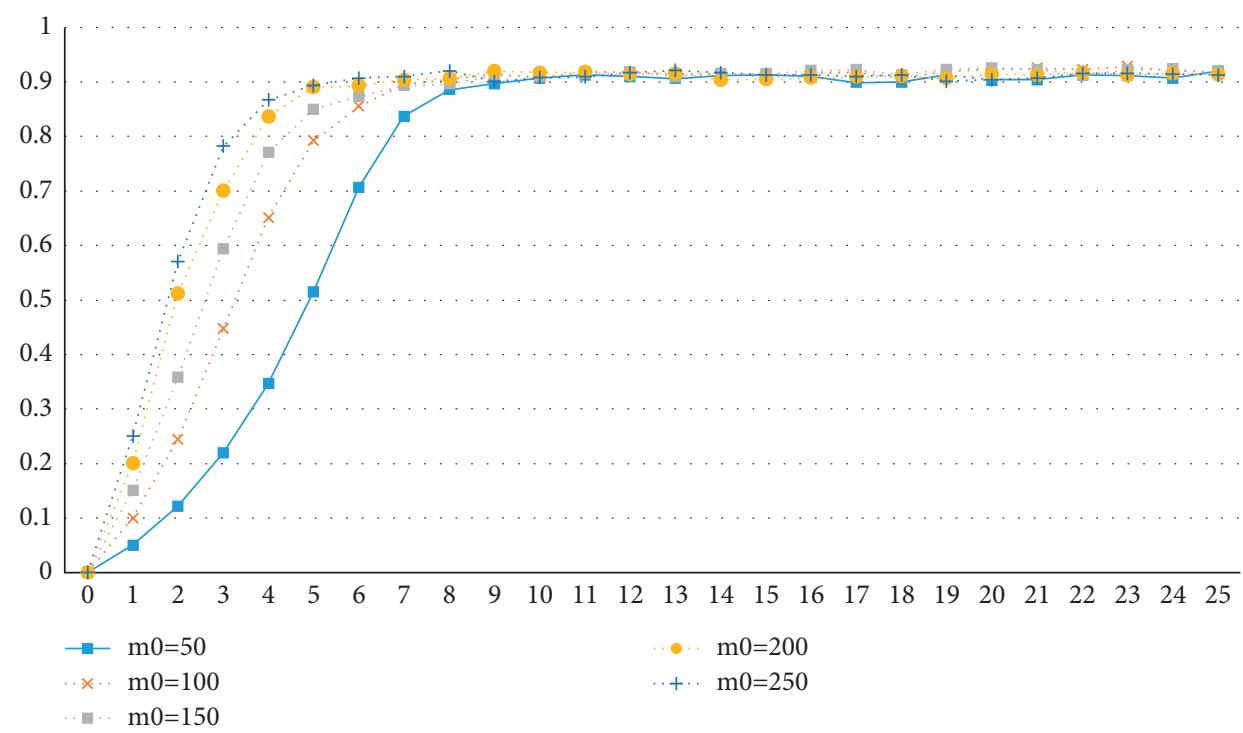

(b)

Figure 2: Effect of initial number of infected nodes $m_{0}$ on $\rho_{k}$. (a) $m_{0}$ ranges from 2 to 10; step length is 2. (b) $m_{0}$ ranges from 50 to 250 ; step length is 50 .

and finally stopped infection. When $m=2$, the contagion rate of credit risk is also low, but it still reaches steady state after 50 steps. This experimental result shows that network complexity is positively correlated with the transmission speed of credit risk. Although network complexity improves the efficiency of supply chain finance to some extent, when confronted with external shocks, more connections between enterprises become the channel of credit risk transmission, thus accelerating the transmission of credit risk.

Next, we discuss the impact of network size on credit risk contagion, and the simulation results are shown in Figure 6. We find that network size has no significant influence on the final steady state: at different scales, network size does not have a significant influence on the steady state, and this conclusion is still valid under the circumstance of increasing external shocks. When the initial shock is small, the network size has a certain impact on the transmission speed of credit risk. When the scale is large, the transmission speed of credit risk is relatively slow. However, with the increasing external shock, the curve highly overlaps, and the impact of network size can be almost ignored.

4.2.3. Analysis of Simulation Results of Policy Intervention Mode under Epidemic Impact. In order to better study the influence of policy intervention on credit risk contagion, the enterprise recovery time $t_{d}$ is introduced into the network to represent the time step required for an enterprise to recover from an infected state to a healthy state under policy intervention. The smaller $t_{d}$ is, the stronger the policy 


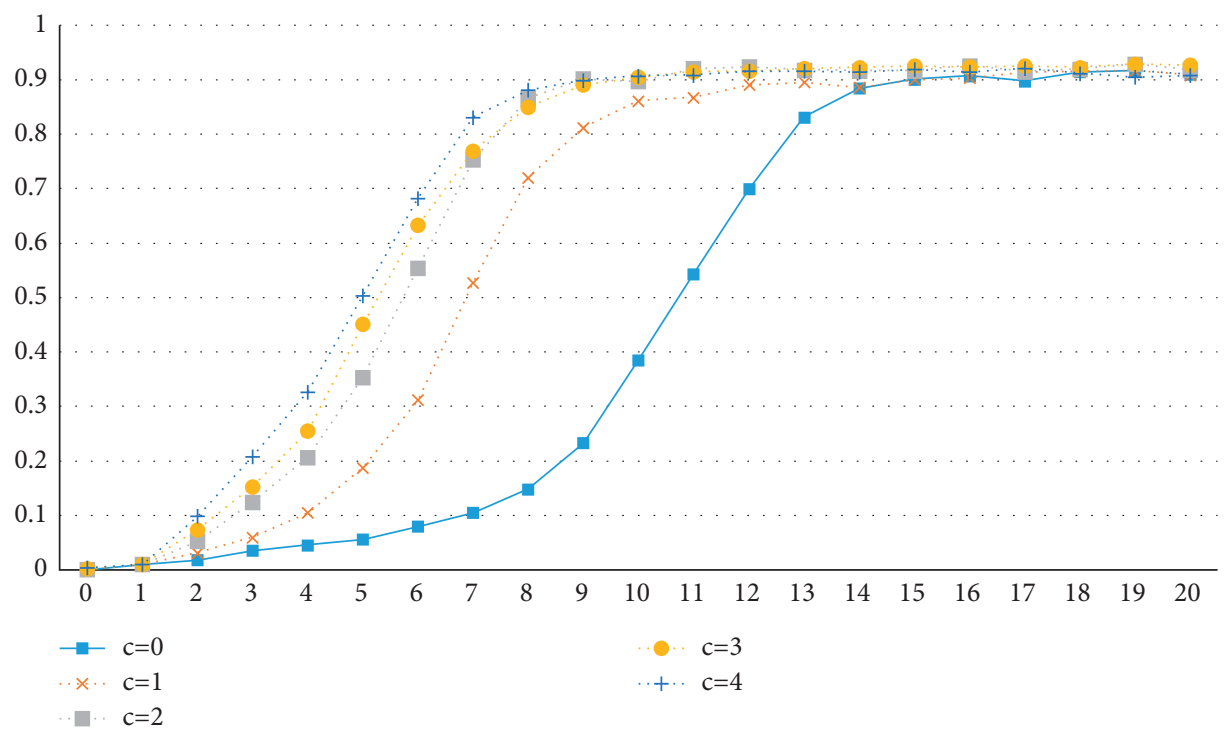

(a)

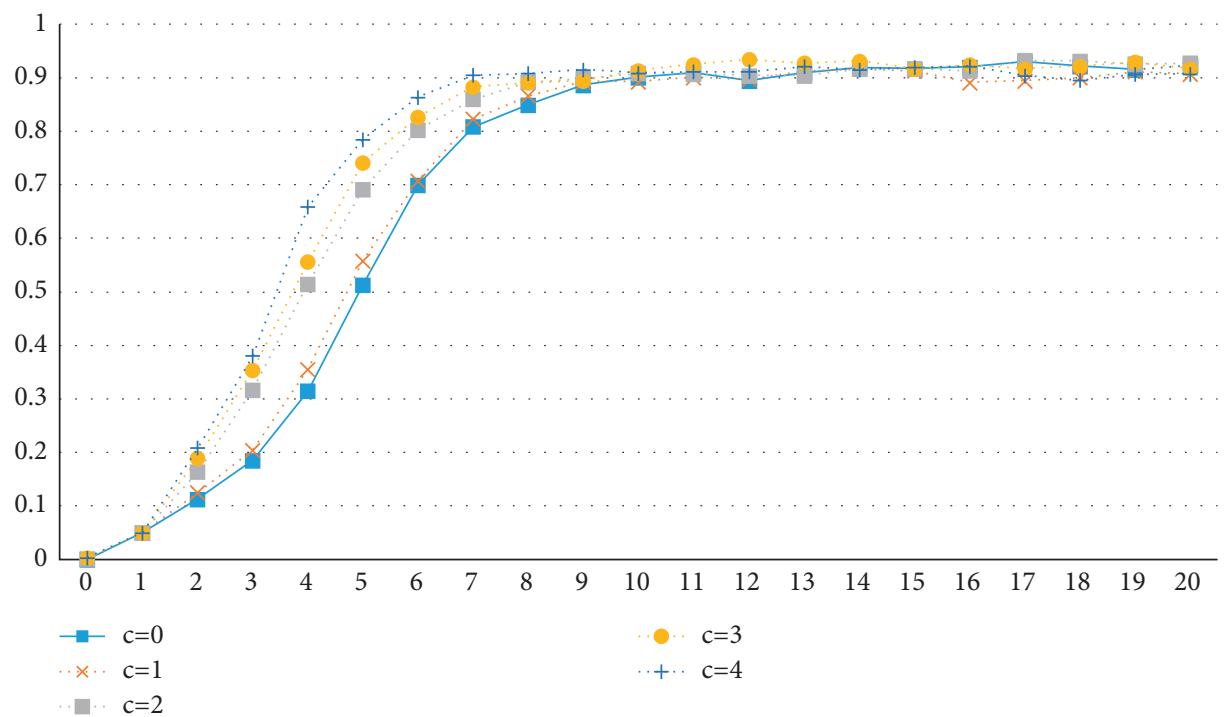

(b)

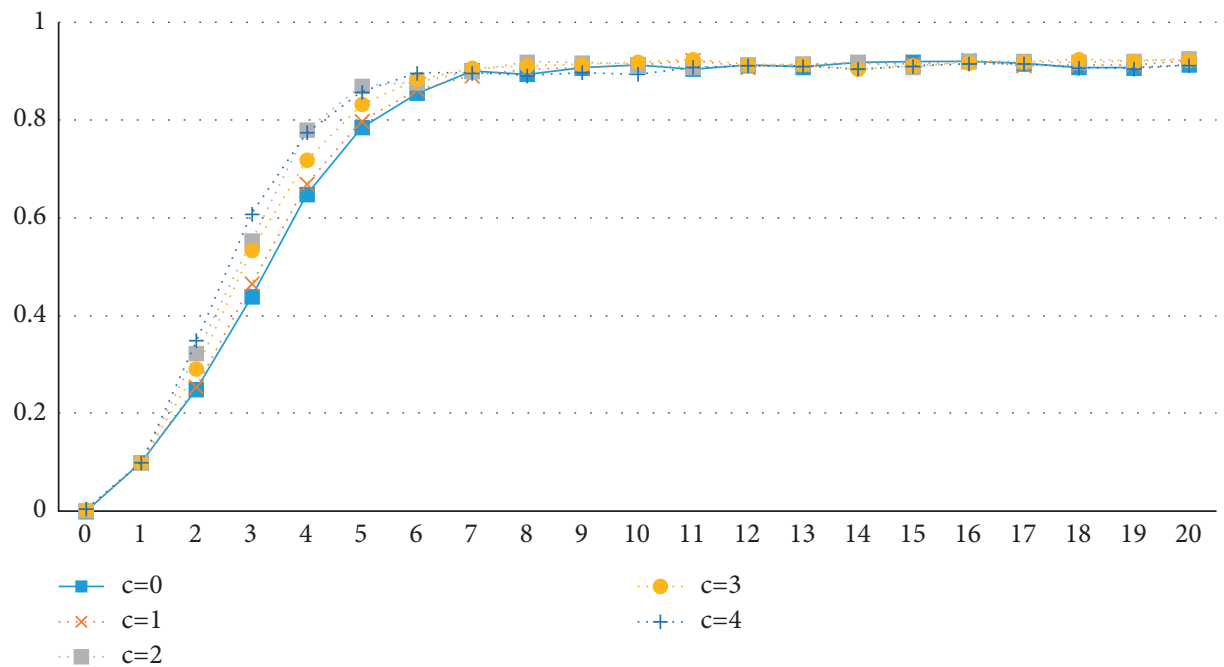

(c)

FIGURE 3: Effect of infected core enterprises $c$ on $\rho_{k}$. (a) $m_{0}=10 ; c$ ranges from 0 to 4 ; step length is 1 . (b) $m_{0}=50$; $c$ ranges from 0 to 4 ; step length is 1 . (c) $m_{0}=100 ; c$ ranges from 0 to 4 ; step length is 1 . 


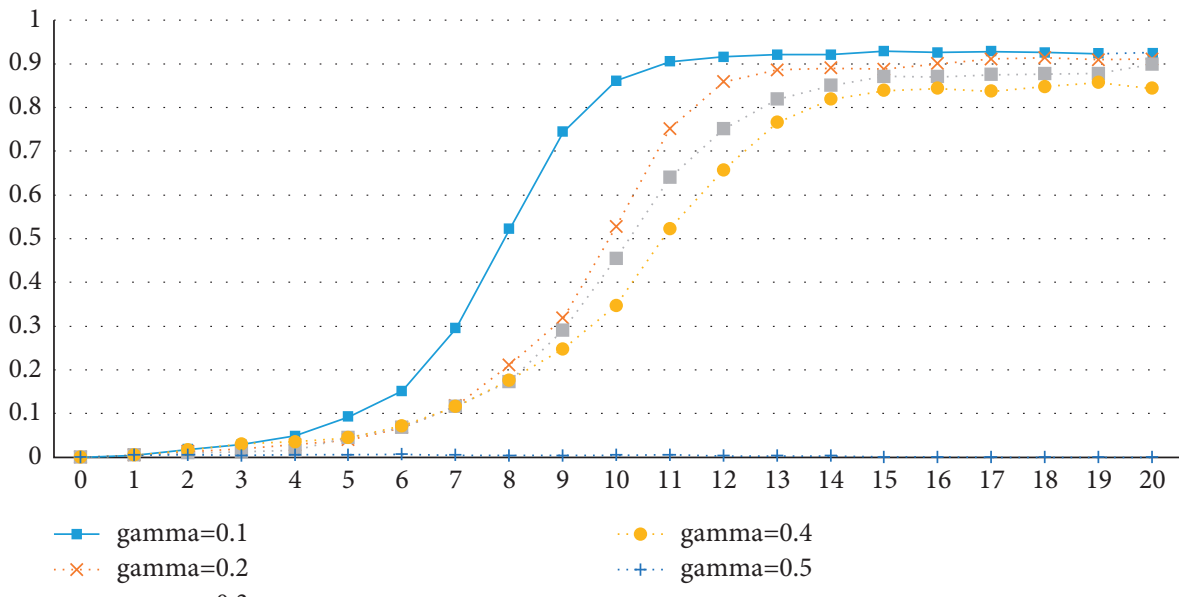

(a)

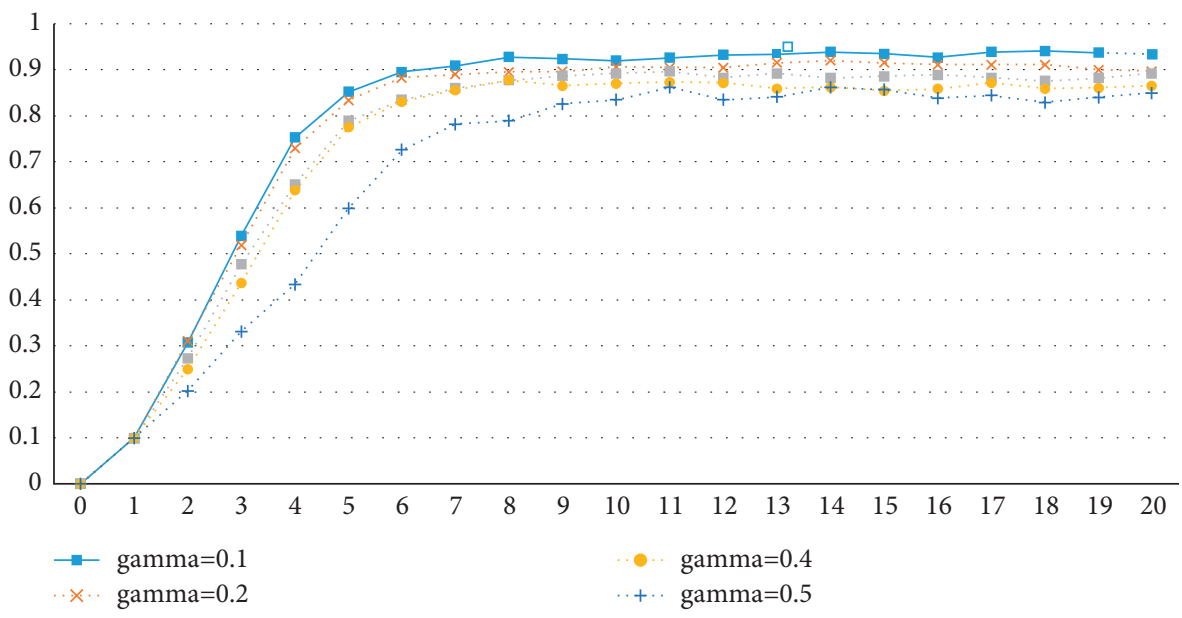

(b)

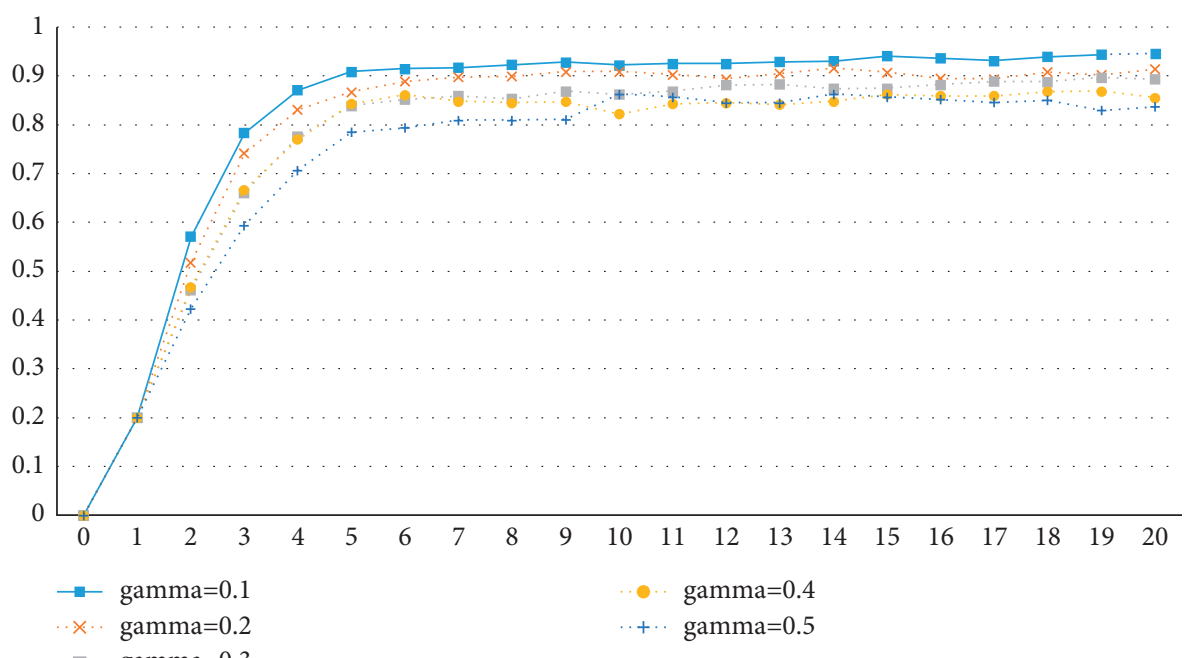

(c)

FIgURE 4: The impact of enterprise self-healing rate $\gamma$ on $\rho_{k}$. (a) $m_{0}=5 ; \gamma$ ranges from 0.1 to 0.5 ; step length is 0.1 . (b) $m_{0}=100 ; \gamma$ ranges from 0.1 to 0.5 ; step length is 0.1 . (c) $m_{0}=200 ; \gamma$ ranges from 0.1 to 0.5 ; step length is 0.1 . 


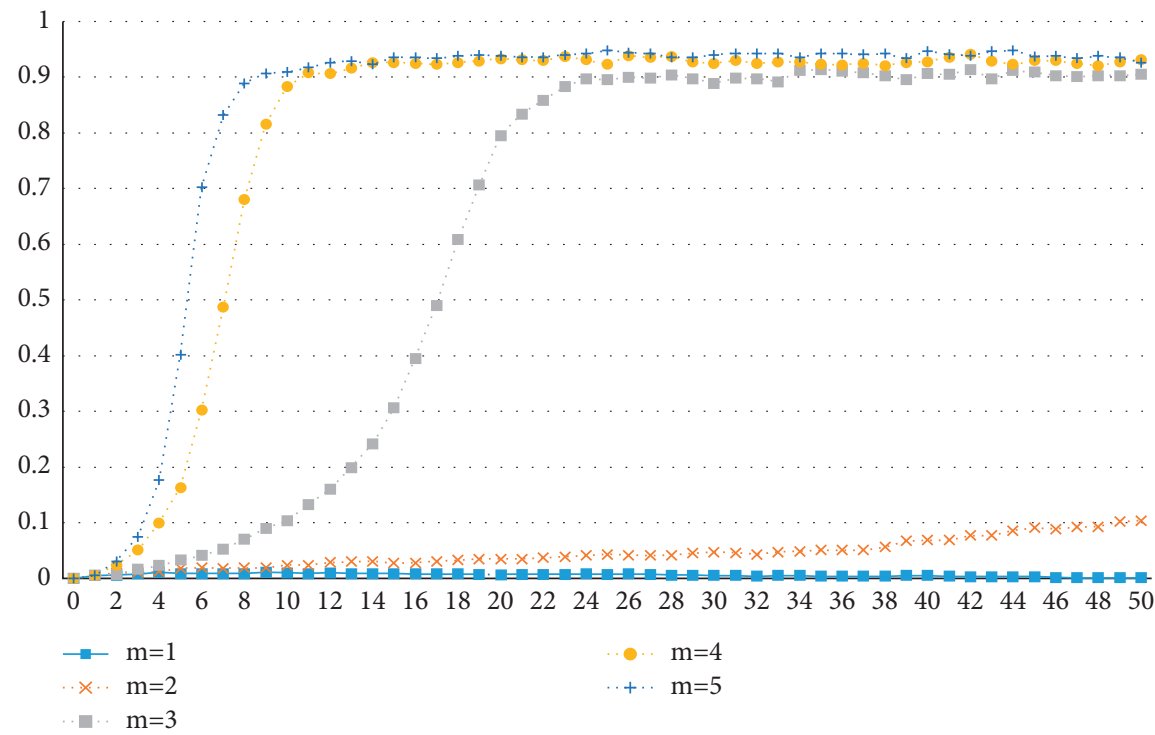

(a)

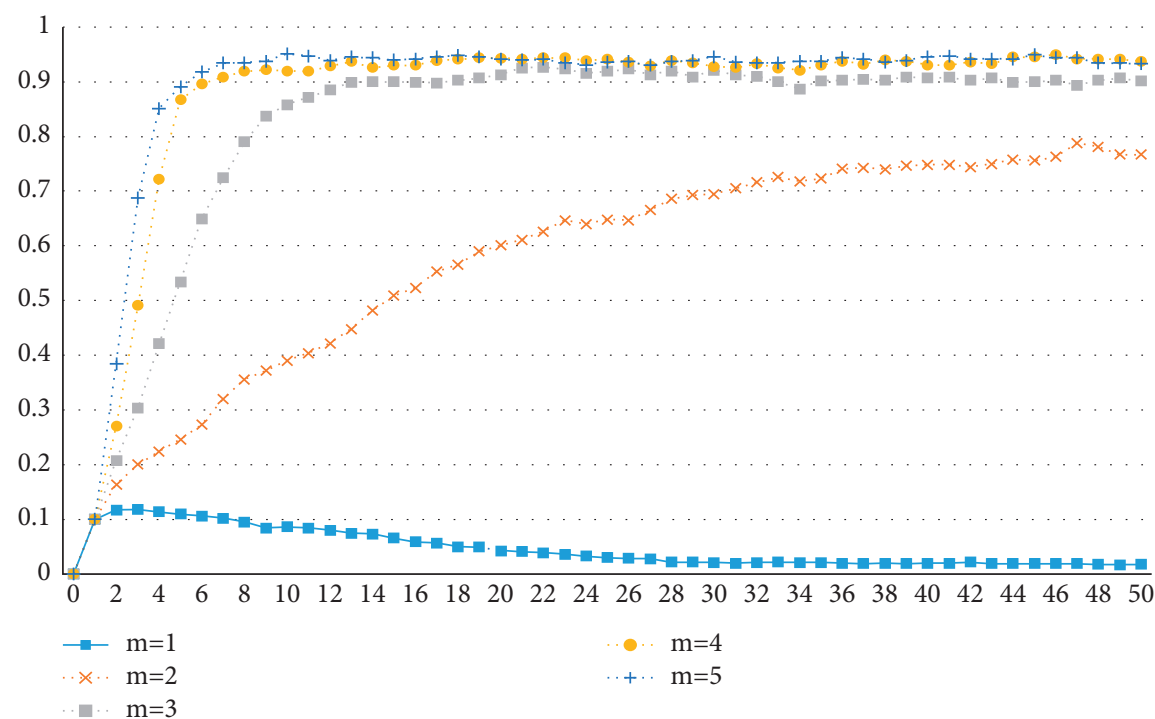

(b)

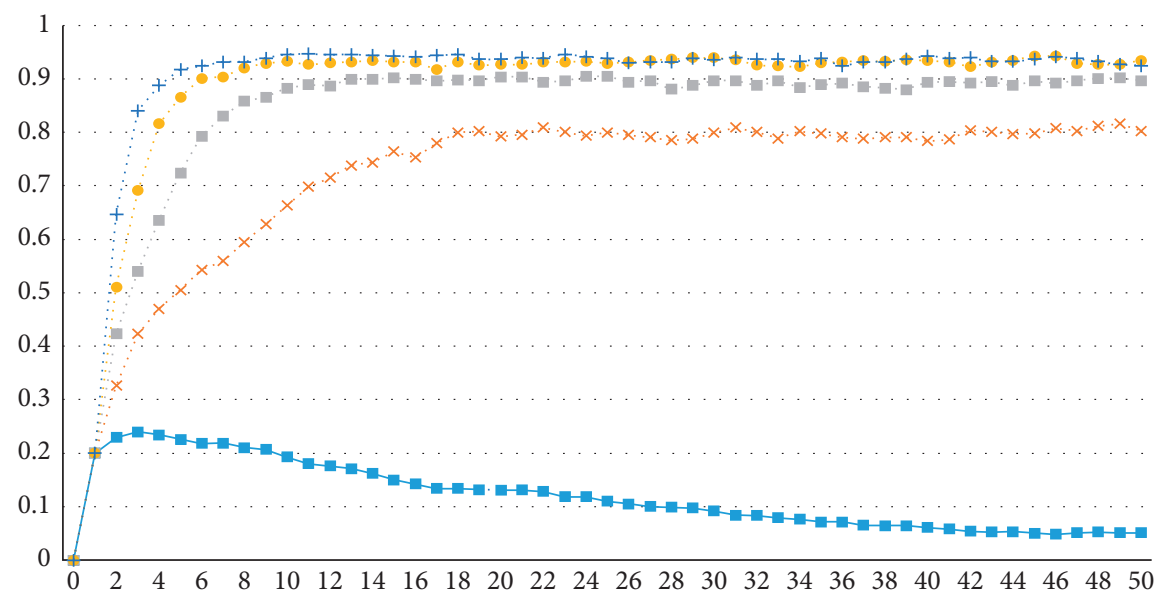

$\rightarrow \mathrm{m}=1$

*.. $\mathrm{m}=2$

-. $\mathrm{m}=4$

-1... $\mathrm{m}=3$

(c)

FiguRE 5: Impact of network complexity $m$ on $\rho_{k}$. (a) $m_{0}=5 ; m$ ranges from 1 to 5 ; step length is 1 . (b) $m_{0}=100$; $m$ ranges from 1 to 5 ; step length is 1 . (c) $m_{0}=200 ; m$ ranges from 1 to 6 ; step length is 1 . 


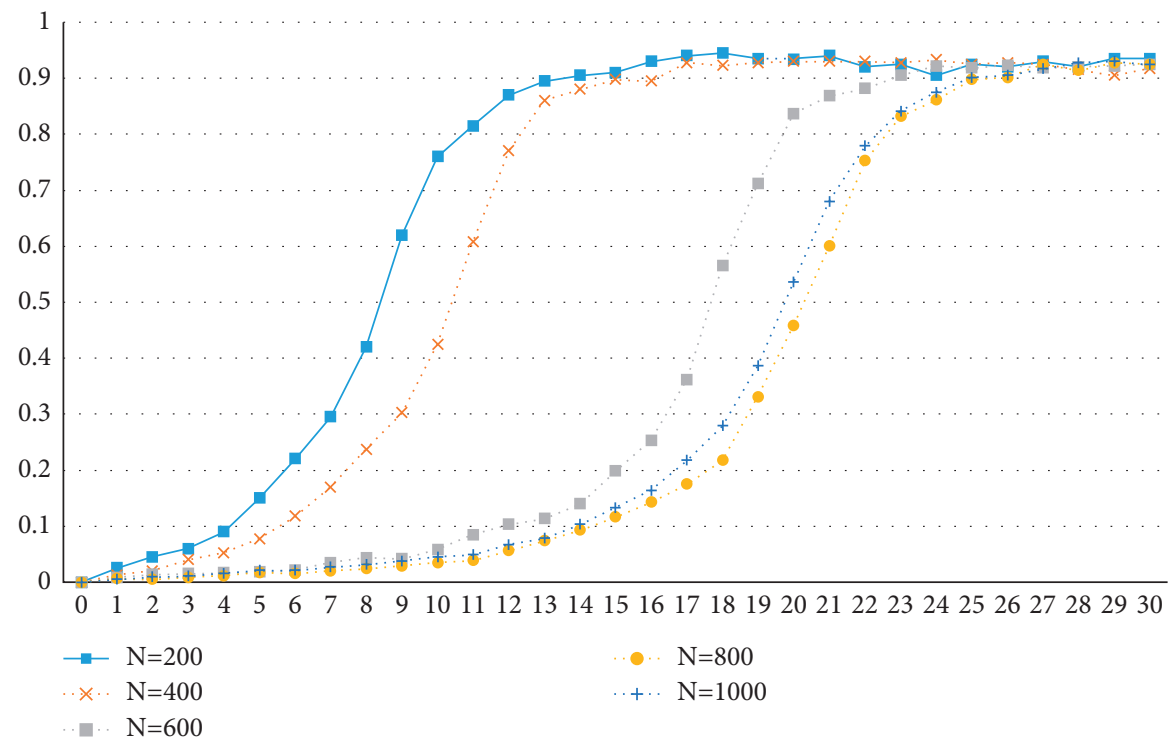

(a)

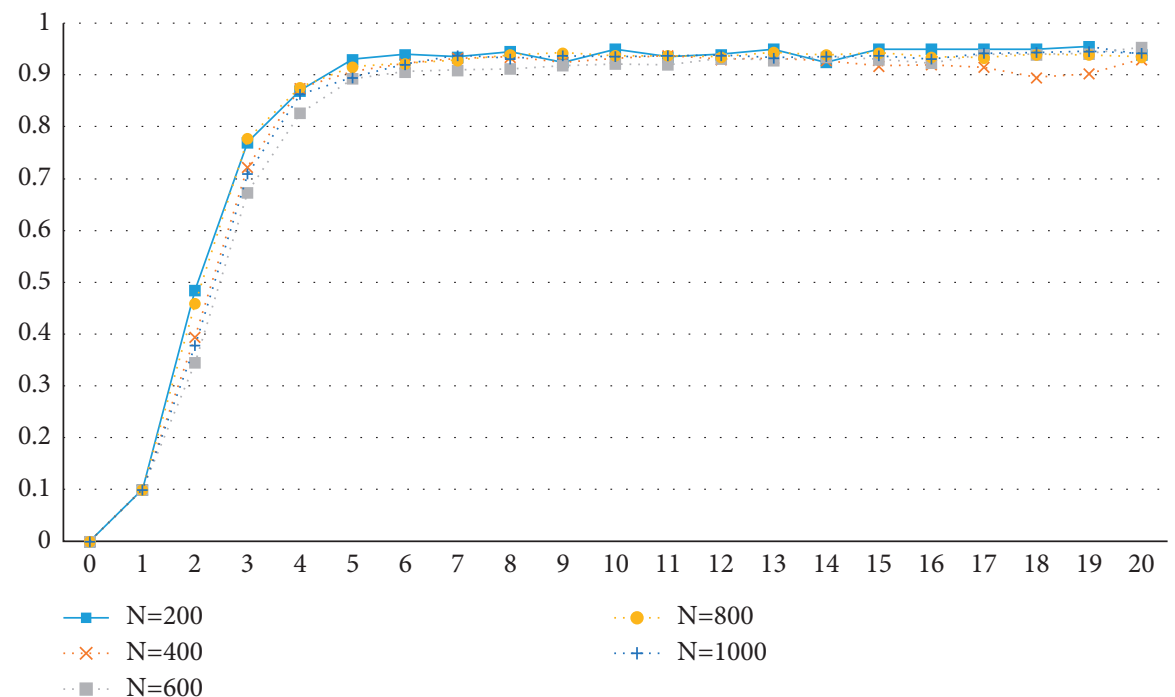

(b)

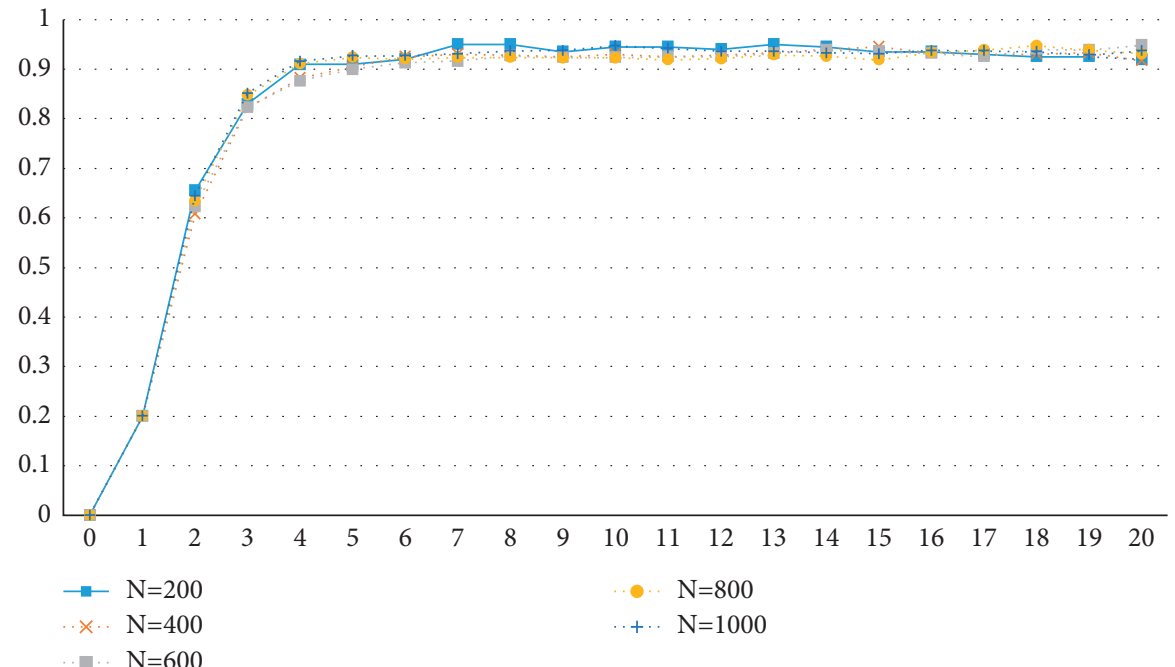

(c)

FiguRE 6: Impact of network size $N$ on $\rho_{k}$. (a) $m_{0}=5 ; n$ ranges from 100 to 1000 ; step length is 200 . (b) $m_{0}=100 ; n$ ranges from 100 to 1000 ; step length is 200. (c) $m_{0}=200 ; n$ ranges from 100 to 1000 ; step length is 200 . 


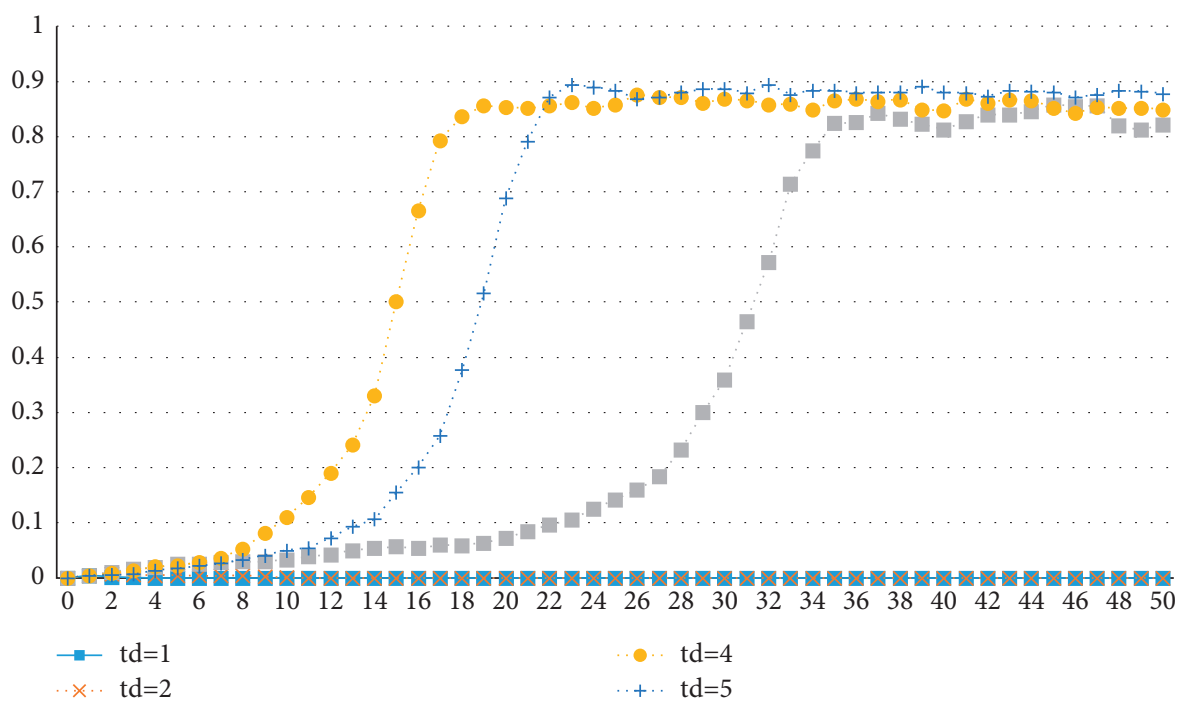

(a)

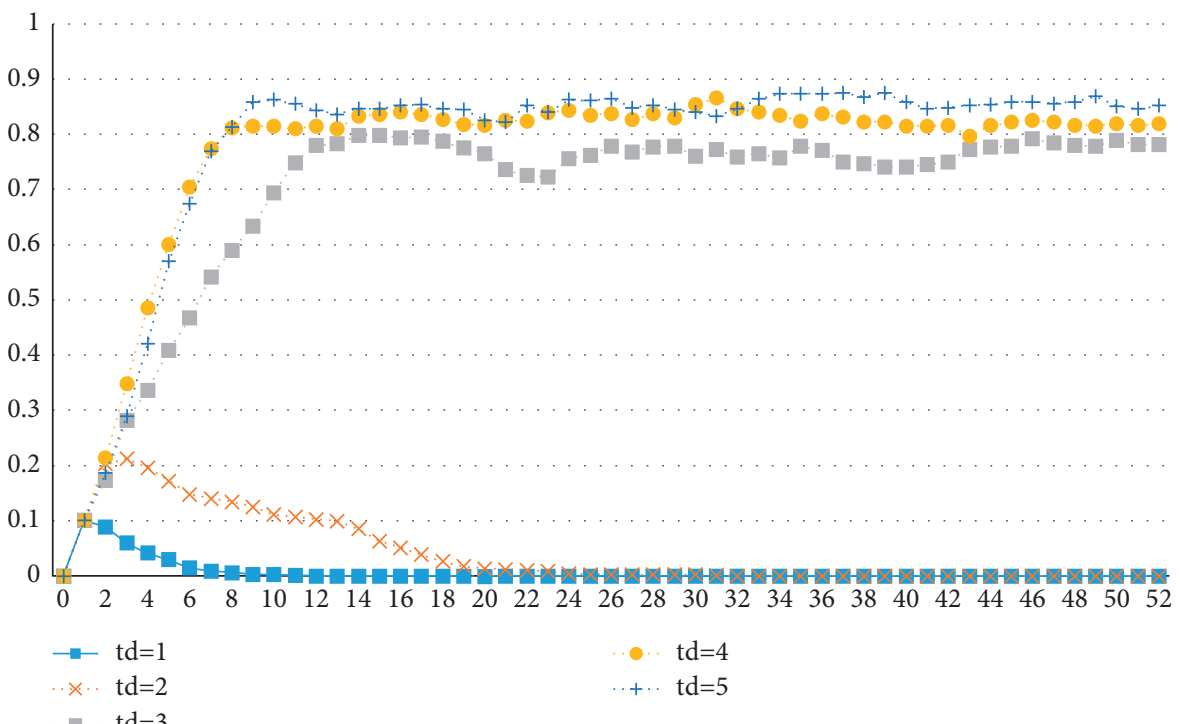

(b)

Figure 7: Impact of policy intervention $t_{d}$ on $\rho_{k}(c=0)$. (a) $m_{0}=5$; $t_{d}$ ranges from 1 to 5 ; step length is 1 . (b) $m_{0}=100 ; t_{d}$ ranges from 1 to 5 ; step length is 1 .

intervention is; otherwise, the weaker it is. We defined $t_{d} \leq 2$ as a strong intervention, $t_{d}=3$ as a moderate intervention, and $t_{d} \geq 4$ as a weak intervention. First of all, we consider the suppression effect of policy intensity on epidemic impact when there is no initial infection of core enterprises $(c=0)$, and the simulation results are shown in Figure 7.

According to the simulation results, we find that in the face of different degrees of external shocks, policy intervention has a significant restraining effect on the rate of credit risk contagion: when the external shocks are small $\left(m_{0}=5\right), t_{d} \leq 2$ can effectively suppress the credit risk contagion. Even if the policy intervention is weak $\left(t_{d} \geq 3\right)$, the contagion rate of credit risk is still low in the initial stage. When the initial impact is enhanced, the inhibitory effect of strong intervention on the epidemic impact is still relatively obvious, but the inhibitory effect of $t_{d} \geq 3$ on the transmission rate of credit risk is weakened. When $m_{0}=100, t_{d}=$ 3 could not completely inhibit the transmission of credit risk. There are two possible explanations for the above conclusions: first, the impact of the epidemic on different industries is different and the intervention intensity is different; second, different levels of intervention are required at different stages of the epidemic. Therefore, the intensity of policy intervention in different industries should be different, and the effect of intervention in the early stage of the outbreak is better, and the cost of intervention is lower. Next, we consider the case of $c=1$, and the simulation results are shown in Figure 8.

The infection of core enterprises has a great impact on the network. In the case of $m_{0}=5, t_{d}<3$ can also restrain the 


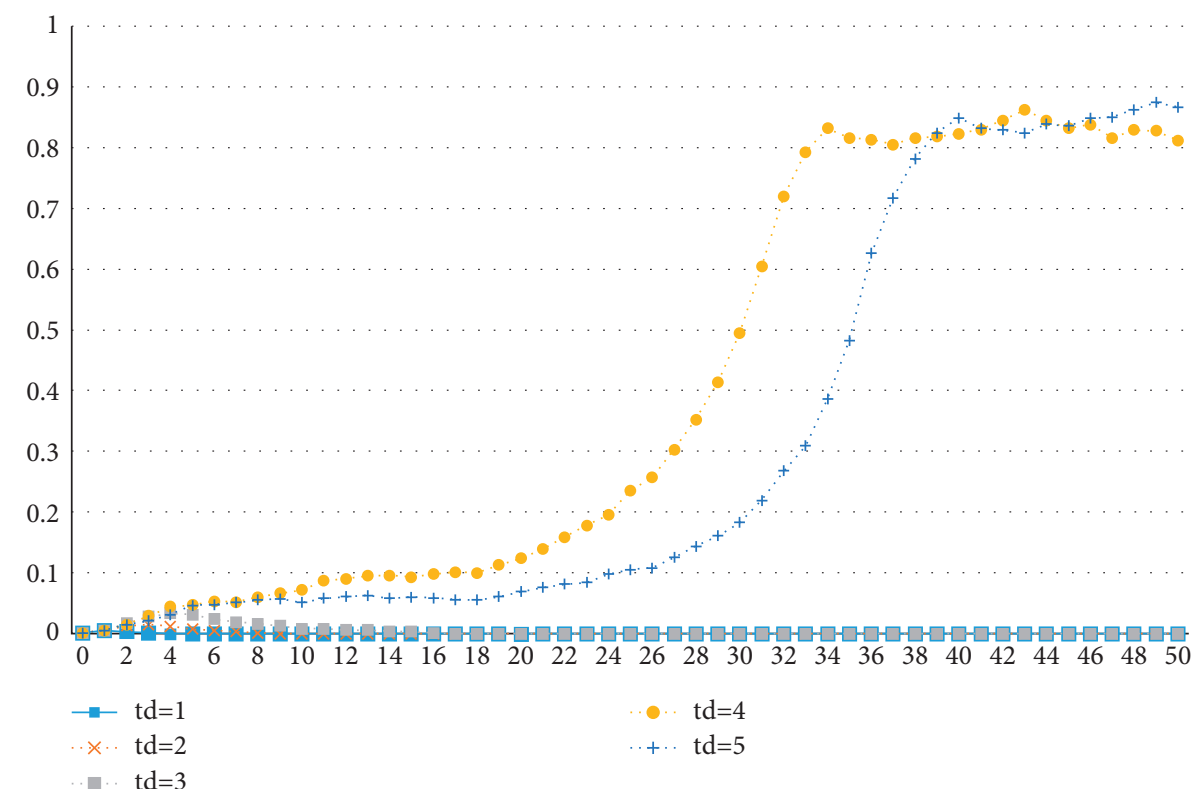

(a)

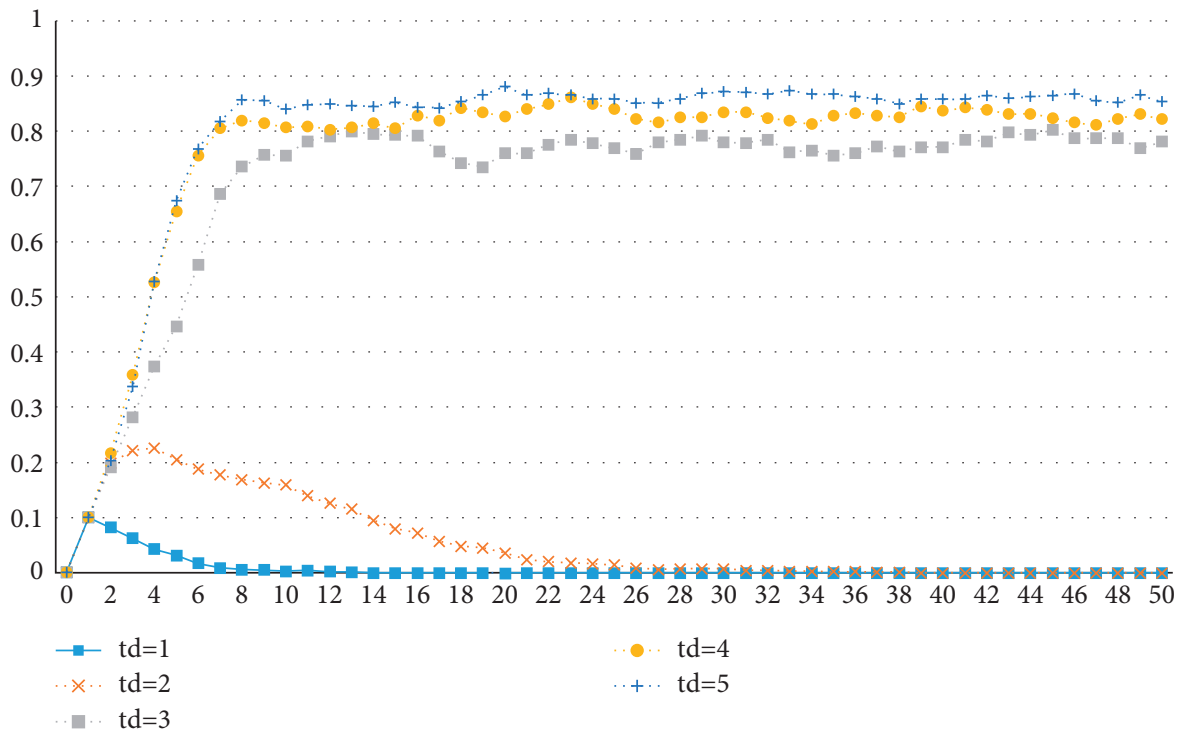

(b)

FIGURE 8: Impact of policy intervention $t_{d}$ on $\rho_{k}(c=1)$. (a) $m_{0}=5$; $t_{d}$ ranges from 1 to 5 ; step length is 1 . (b) $m_{0}=100$; $t_{d}$ ranges from 1 to 5 ; step length is 1 .

credit risk contagion. However, with the strengthening of external shocks $\left(m_{0}=100\right), t_{d}=3$ is unable to restrain the credit risk contagion, and the credit risk contagion speed increases greatly when $t_{d} \geq 4$. Due to the importance of the health status of core enterprises to the network risk transmission, attention should be paid in the process of the outbreak.

\section{Conclusion}

This paper conducts a simulation experiment on the credit risk contagion of supply chain finance network under the COVID-19 pandemic. First, considering the mode of self- healing without policy intervention, we find that the increase in the initial number of infected nodes will significantly increase the contagion rate of credit risk, but the impact on the steady state is relatively limited. The self-healing rate of enterprises can significantly reduce the speed of credit risk contagion and the density of infected nodes in the steady state. As the complexity of network increases the channels of credit risk transmission, the speed of risk transmission increases. However, the network size has no significant influence on the infection rate and the steady state. However, when the external shock is large enough, the steady state of the network is above $80 \%$, which indicates that in the absence of policy intervention, the self-healing ability of 
enterprises alone cannot resist the impact of external shock on the network, resulting in the failure of the supply chain finance network to play its due function.

Second, under the external shock, policy intervention is essential to ensure the normal operation of the supply chain and reduces the possibility of credit risk contagion. In the case of small external impact, even moderate intensity intervention can achieve the effect of infection suppression. However, as external shocks grow, so too should the scale of intervention. This shows that the impact of the epidemic on different industries is different, and policy intervention measures should also be targeted. In the early stage of the epidemic, timely and targeted government intervention will achieve obvious results.

Finally, due to the importance of its position, the speed of the credit risk contagion will be significantly increased once credit default occurs in core enterprises of the supply chain. Therefore, whilst focusing on key industries and preventing the supply chain network from being severely impacted, the health of core enterprises should be focused on.

This paper has some limitations and could be improved in the follow-up research. First of all, this paper ignores the particularity of different supply chain financial networks and selects the same contagion probability. Subsequent studies can further explore the characteristics of specific networks. In addition, this paper assumes that the scale of the network remains unchanged and does not consider the entry and exit mechanism of nodes. In order to be closer to the reality, corresponding discussions can be made in subsequent studies.

\section{Data Availability}

The data used to support the findings of this study are available from the corresponding author upon request.

\section{Conflicts of Interest}

The authors declare that they have no conflicts of interest.

\section{References}

[1] S. Y. Cho, "A convergence theorem for generalized mixed equilibrium problems and multivalued asymptotically nonexpansive mappings," Journal of Nonlinear Convex Analysis, vol. 21, pp. 1017-1026, 2001.

[2] D. R. Sahu, J. C. Yao, M. Verma, and K. K. Shukla, "Convergence rate analysis of proximal gradient methods with applications to composite minimization problems," Optimization, vol. 70, no. 1, pp. 75-100, 2021.

[3] S. Cho, "A monotone Bregan projection algorithm for fixed point and equilibrium problems in a reflexive Banach space," Filomat, vol. 34, no. 5, pp. 1487-1497, 2020.

[4] G. Colajanni, P. Daniele, and D. Sciacca, "A projected dynamic system associated with a cybersecurity investment model with budget constraints and fixed demands," Journal of Nonlinear Variational Analysis, vol. 4, pp. 45-61, 2019.

[5] N. T. An, "Robust feature selection via nonconvex sparsitybased methods," Journal of Nonlinear and Variational Analysis, vol. 5, no. 1, pp. 59-77, 2021.
[6] S. Carnovale, D. S. Rogers, and S. Yeniyurt, "Broadening the perspective of supply chain finance: the performance impacts of network power and cohesion," Journal of Purchasing and Supply Management, vol. 25, no. 2, pp. 134-145, 2019.

[7] M. Kamalahmadi and M. M. Parast, "A review of the literature on the principles of enterprise and supply chain resilience: major findings and directions for future research," International Journal of Production Economics, vol. 171, pp. 116-133, 2016.

[8] X. Liu, L. Zhou, and Y.-C. Wu, "Supply chain finance in China: business innovation and theory development," Sustainability, vol. 7, no. 11, pp. 14689-14709, 2015.

[9] W. Mou, W.-K. Wong, and M. McAleer, "Financial credit risk evaluation based on core enterprise supply chains," Sustainability, vol. 10, no. 10, p. 3699, 2018.

[10] H. Xu, "Minimizing the ripple effect caused by operational risks in a make-to-order supply chain," International Journal of Physical Distribution \& Logistics Management, 2020.

[11] J. Wang, H. Zhou, and X. Jin, "Risk transmission in complex supply chain network with multi-drivers," Chaos, Solitons \& Fractals, vol. 143, p. 110259, 2021.

[12] H.-C. Pfohl and M. Gomm, "Supply chain finance: optimizing financial flows in supply chains," Logistics Research, vol. 1, no. 3-4, pp. 149-161, 2009.

[13] E. Hofmann, "Supply Chain Finance-some conceptual insights,” Logistics Management, pp. 203-214, 2005.

[14] F. Caniato, L. M. Gelomino, A. Perego, and S. Ronchi, "Does finane solve the supply chain financing problem?" Supply Chain Management: International Journal, vol. 21, p. 5, 2016.

[15] Y. Zhu, L. Zhou, C. Xie, G.-J. Wang, and T. V. Nguyen, "Forecasting SMEs' credit risk in supply chain finance with an enhanced hybrid ensemble machine learning approach," International Journal of Production Economics, vol. 211, pp. 22-33, 2019.

[16] K. Wang, F. Yan, and Y. Zhang, "Supply chain financial risk evaluation of small-and medium-sized enterprises under smart city," Journal of Advanced Transportation, vol. 2020, Article ID 8849356, 14 pages, 2020.

[17] Z. Zhao, D. Chen, L. Wang, and C. Han, "Credit risk diffusion in supply chain finance: a complex networks perspective," Sustainability, vol. 10, no. 12, p. 4608, 2018.

[18] X. Xie, Y. Yang, J. Gu, and Z. Zhou, "Research on the contagion effect of associated credit risk in supply chain based on dual-channel financing mechanism," Environmental Research, vol. 184, Article ID 109356, 2020.

[19] K. Yang and Z. Zhang, "Simulation of SIS-RP model in supply chain network risk propagation," Journal of Beijing Jiaotong University, vol. 37, no. 3, pp. 122-126, 2013.

[20] W. Wang and W. P. Fu, "Review of research on modeling and simulation for dynamics and complexity of supply chain systems," Journal of System Simulation, vol. 2, 2010. 